\title{
PRODUCT RECURRENCE AND DISTAL POINTS
}

\author{
J. AUSLANDER AND H. FURSTENBERG
}

\begin{abstract}
Recurrence is studied in the context of actions of compact semigroups on compact spaces. (An important case is the action of the Stone-Čech compactification of an acting group.) If the semigroup $E$ acts on the space $X$ and $F$ is a closed subsemigroup of $E$, then $x$ in $X$ is said to be $F$ recurrent if $p x=x$ for some $p \in F$, and product $F$-recurrent if whenever $y$ is an $F$-recurrent point (in some space $Y$ on which $E$ acts) the point $(x, y)$ in the product system is $F$-recurrent. The main result is that, under certain conditions, a point is product $F$-recurrent if and only if it is a distal point.
\end{abstract}

\section{INTRODUCTION}

Recurrence is a form of asymptotic behavior for a dynamical system; namely behavior that relates to the action of group elements going out to infinity. In the classical case where the acting group is one dimensional there are just two directions in which an element can go out to infinity, and asymptotic notions take on a deceptively simplified form. Even here one does distinguish between positive and negative recurrence of points (and these are distinct notions). When the acting group is multidimensional, there are many more possibilities and we may study a variety of notions of recurrence. Having done this, we will see that the one dimensional situation too is richer than it appears on the surface.

Classically, a topological dynamical system consists of a topological space $X$ (we take it to be metric) with a one-parameter group $\left\{T_{t}\right\}_{t \in R}$ or $\left\{T^{n}\right\}_{n \in \mathbf{Z}}$ of homeomorphisms acting on it. We say $x_{0} \in X$ is recurrent if for some $t_{k} \rightarrow \pm \infty$ (or $n_{k} \rightarrow \pm \infty$ ) $T_{t_{k}} x_{0} \rightarrow x_{0}$ (or $T^{n_{k}} x_{0} \rightarrow x_{0}$ ). We can distinguish between positive and negative recurrence according as the group elements converge to $+\infty$ or $-\infty$. Whenever a point is recurrent, we find that there is actually a richer structure for the set of (say) $n_{k}$ with $T^{n_{k}} x_{0} \rightarrow x_{0}$. For, by choosing $n_{i}$ with $T^{n_{i}} x_{0} \rightarrow x_{0}$ sufficiently rapidly, we will also have $T^{n_{i}+n_{j}+\cdots+n_{l}} x_{0}$ close to $x_{0}$ for $i<j<\cdots<l$ provided $i$ is sufficiently large. The sets of sums $\left\{n_{i}+n_{j}+\cdots+n_{l}\right\}$ for $i<j<\cdots<l$ is what is called an $I P$-system (in $\mathbb{Z}$ ). We abbreviate $n_{\alpha}=n_{i}+n_{j}+\cdots+n_{l}$ where $\alpha$ is the multi-index $\alpha=\{i, j, \ldots, l\}$, a finite subset of $\mathbb{N}$. We say $\alpha \rightarrow \infty$ if $\min _{i \in \alpha} i \rightarrow \infty$, and we obtain " $I P$ recurrence": $T^{n_{\alpha}} x_{0} \rightarrow x_{0}$, as $\alpha \rightarrow \infty$ (see [3] for details regarding $I P$-systems). It becomes natural to treat recurrence in the framework of IP-subsystems of

Received by the editors February 26, 1992 and, in revised form, July 10, 1992.

1991 Mathematics Subject Classification. Primary 54H20. 
a group. We can now obtain a strengthened version of recurrence by insisting that one has recurrence along all $I P$-subsystems of a given system. One reason for interest in this stems from the fact that if we require $I P$-recurrence for all $I P$-systems in the group, then the corresponding notion of recurrence for a point $x_{0}$ is equivalent to that of distality (see [3]). This in turn implies not only that $x_{0}$ is recurrent, but if we consider product systems $\left(X \times Y, T^{n} \times S^{n}\right)$, then for any recurrent point $y_{0} \in Y$ the pair $\left(x_{0}, y_{0}\right)$ is again recurrent. It is easy to give examples of points which are individually recurrent, but not jointly so. (For example, $x_{0}$ might be positively recurrent and $y_{0}$ negatively recurrent.) Thus we are led to a notion of product recurrence.

An alternative way of dealing with the "direction" of recurrence is to introduce the Stone-Čech compactification of the acting group. Call the group $G$ and its Stone-Čech compactification $\beta G . \quad \beta G$ is a (noncommutative) semigroup, and when $G$ acts on a topological space $X$, there is a naturally induced action of $\beta G$ on $X$. Sequences of group elements tending out to infinity in $G$ cluster around points of $\beta G \backslash G$. The cluster points for an $I P$-subsystem in $G$ form a closed semigroup, say $F \subset \beta G \backslash G$. If $x_{0}$ is a recurrent point for an $I P$-sequence clustering at $F$ then we will have $p x_{0}=x_{0}$ for some $p \in F$. More generally, if $F$ is any closed semigroup in $\beta G$, we may speak of $F$ recurrence of $x_{0}$ when $p x_{0}=x_{0}$. In analogy with the previous paragraph, we may also speak of product $F$-recurrence of $x_{0}$ : for every $F$-recurrent point $y$ (in any system $(Y, G))$ the point $\left(x_{0}, y\right)$ is an $F$-recurrent point of the product system. While $F$-recurrence becomes a weaker property as $F$ is enlarged, under appropriate hypotheses product $F$-recurrence turns out to be a stronger property the larger $F$ is taken. One should think of product $F$-recurrence as recurrence in all directions ending in $F$. As we shall see, as soon as $F$ is taken sufficiently large, product $F$-recurrence becomes independent of $F$ and is equivalent to distality. In the case of $\mathbb{Z}$ we may take $F$ either as $\operatorname{cl}\left(\mathbb{Z}^{-}\right)$ or $\operatorname{cl}\left(\mathbb{Z}^{+}\right)$and we obtain the same notion of product $F$-recurrence (but not of $F$-recurrence). This is not a transparent consequence of the definition.

The approach we take in this paper is based on these considerations. We treat directly the actions of a compact semigroup $E$ and its closed subsemigroups $F \subset E$. We have in mind principally the example of the Stone-Čech compactification of a group. But we also consider an example which deals directly with the natural " $I P$-structure" implicit in recurrence and take the acting system to be not a group, but the family $\mathscr{F}$ of finite subsets of $\mathbb{N}$. Ellis' theory of enveloping semigroups for dynamical systems will be the inspiration for our discussion, with idempotents playing a key role. One novelty that appears in our discussion is that here "maximal" idempotents will play an important part. We are not aware that the significance of maximal idempotents has been noted previously.

Finally we point out that there is another example of an asymptotic notion in both topological and measurable dynamics in which the one-dimensional case disguises the more complex structure, and that is the notion of mixing. Here we cannot distinguish between positive and negative mixing, but it does make sense to speak of mixing along an $I P$-system. One has yet to develop systematically the notion of " $F$-mixing". But we can note that "product $F$-mixing" when $F=\beta G \backslash G$ is just the notion of mild mixing treated in [4 and 5]. 


\section{RECURRENCE AND PRODUCT RECURRENCE}

We consider nonempty compact Hausdorff spaces $E$ equipped with a semigroup structure such that the maps $p \rightarrow p q$ are continuous. Let $\mathscr{E}$ be the class of such semigroups. If $E \in \mathscr{E}$, by an action of $E$ on a compact Hausdorff space $X$ we mean a map from $E \times X$ to $X((p, x) \rightarrow p x)$ such that $p(q x)=(p q) x(p, q \in E, x \in X)$ and such that the maps $p \rightarrow p x$ from $E$ to $X$ are continuous. (It is not assumed that the maps $x \rightarrow p x$ are continuous.) It follows that the collection of maps defined by $E$ is a closed subset of $X^{X}$. We call such an action of $E$ on $X$ a flow, which we denote by $(X, E)$.

The standard notions of topological dynamics carry over to such semigroup actions, with little or no change in the proofs. A straightforward Zorn's lemma argument guarantees the existence of minimal sets-subsets $K$ of $X$ which are nonempty, closed, $E$ invariant $(E K \subset K)$ and minimal with respect to these three properties. (The usual assumption of continuity of the maps $x \rightarrow p x$ is not necessary for the proof.) A point on a minimal set is called an almost periodic point. If $K$ is a minimal set, then $E x=K$, for every $x \in K$.

If $E \in \mathscr{E}, E$ acts on itself by left multiplication; in the above terminology $(E, E)$ is a flow. The minimal sets for this action are precisely the minimal left ideals of the semigroup $E$ (that is, nonempty subsets $I$ of $E$ such that $E I \subset I$, which are minimal with respect to these properties). It follows that minimal left ideals are necessarily closed. Moreover, if $(X, E)$ is a flow, and $I$ is a minimal left ideal in $E$, then $I X$ is a minimal set and every minimal set in $X$ is of this form.

We will make repeated use of a fundamental result of Ellis-if $E \in \mathscr{E}$ then $E$ contains an idempotent $[1,2]$. Let $J=J(E)$ denote the set of idempotents in $E$. We define a quasi order (a reflexive, transitive relation) $>$ in $J$ by $u>v$ if $u v=v$. If $u>v$ and $v>u$ we say that $u$ and $v$ are equivalent and write $u \approx v$. It is easily verified that $>$ is a quasi order, (and therefore that $\approx$ is an equivalence relation). The quasi order $>$ on $J$ may be regarded as being defined on equivalence classes. That is, if $u, v, u^{\prime}, v^{\prime} \in J$ with $u>v, u^{\prime} \approx u, v^{\prime} \approx v$, then $u^{\prime}>v^{\prime}$, since $u^{\prime}>u>v>v^{\prime}$.

An $m \in J$ will be called maximal if whenever $n \in J$ with $n>m$, then $n \approx m$; minimal idempotents are defined similarly.

If $E \in \mathscr{E}$, and $I$ is a minimal left ideal in $E$, then $I \in \mathscr{E}$, so $I$ contains idempotents. Moreover, the equivalence relation $\approx$ is the same as the equivalence relation defined by Ellis for idempotents in minimal left ideals. If $I$ and $I^{\prime}$ are minimal left ideals in $E$, and $u$ is an idempotent in $I$, then there is a unique idempotent $u^{\prime}$ in $I^{\prime}$ such that $u \approx u^{\prime}$ ([1,2]; however, "right" notation is used in these references). This shows that the quasi order $>$ is in general not a partial order.

Lemma 1. With respect to the quasi order $>, J$ contains maximal and minimal idempotents. If $c \in J$, there are maximal and minimal idempotents $m$ and $u$ such that $m>c>u$. The minimal idempotents are precisely those idempotents which are in some minimal left ideal of $E$.

Proof. To show the existence of maximal idempotents in $J$, let $c \in J$ and let $\left\{c_{i}\right\}$ be a totally ordered family in $J$ with $c_{i}>c$. Regard $\left\{c_{i}\right\}$ as a net, and let (a subnet of) $c_{i} \rightarrow r \in E$. Then for fixed $i$, if $c_{j}>c_{i}, c_{j} c_{i}=c_{i}$, so $r c_{i}=c_{i}$. Let $H=\left[q \in E \mid q c_{i}=c_{i}\right.$, for all $\left.c_{i}\right]$. Then $H$ is a nonempty closed 
semigroup, and hence contains an idempotent $w$. Clearly $w>c_{i}>c$. Thus Zorn's lemma applies and $J$ contains maximal idempotents.

Now let $u$ be an idempotent in a minimal left ideal $I$ of $E$. If $c \in J$ with $u>c$ then $c=u c \in I c=I^{\prime}$, a minimal left ideal and it follows [1,2] that $u \approx c$. Thus every idempotent in a minimal left ideal is also minimal with respect to the quasi order $>$. Now let $c \in J$, and let $I$ be a minimal left ideal contained in $E$. Then $I^{*}=I c$ is a minimal left ideal.

Let $v$ be an idempotent in $I^{*}$. Then $v=k c$, for some $k \in I$. Note that $v c=k c c=k c=v$. Moreover, $c v c v=c v v=c v$ so $c v \in J$ and $c v \in c I^{*} \subset I^{*}$. Also $c c v=c v$ so $c>c v$. If $c$ is already a minimal idempotent with respect to $>, c \approx c v$ and $c=c v c \in I^{*} c$, so $c$ is in a minimal left ideal.

If $E$ contains $e$, a (two sided) identity, then clearly $e$ is the unique maximal element of $J(E)$. The examples of interest to us will in general not contain identity elements.

We now review some basic dynamical notions in the context of flows $(X, E)$ where $E \in \mathscr{E}$. It is easy to see that these coincide with the corresponding notions in the case of actions of a group $G$ on $X$, and $E=\beta G$ (or the enveloping semigroup of the flow $(X, G))$.

If $E \in \mathscr{E}$ acts on $X$, then $x$ and $y$ in $X$ are said to be proximal if $p x=p y$ for some $p$ in $E$. If $x$ and $y$ are not proximal, they are said to be distal. The point $x$ is distal if $x$ and $y$ are distal for all $y \in E x$ with $y \neq x$, and the flow $(X, E)$ is called distal if all points are distal points. If $x \in X, x$ is proximal to an almost periodic point-namely $u x$, where $u$ is a minimal idempotent. It follows that a distal point is an almost periodic point.

If $E \in \mathscr{E}$ is fixed, we will consider closed subsemigroups $F$ of $E$ which do not contain the identity. Let $\mathscr{S}$ denote the collection of such semigroups $F$.

If $E$ acts on $X, F \in \mathscr{S}$, and $x \in X$, we say that $x$ is $F$-recurrent if $r x=x$ for some $r \in F$.

This definition includes the usual notion of (positive) recurrence when the integers $\mathbb{Z}$ or the positive integers $\mathbb{N}$ act on a compact metric space $X$ as a group of homeomorphisms. In this case, $E$ is $\beta \mathbb{Z}$ or $\beta \mathbb{N}$ (the Stone-Čech compactification) and $F=\beta \mathbb{N} \backslash \mathbb{N}$. Also, for any action, if $I$ is a minimal left ideal in $E$, then $I$-recurrence coincides with almost periodicity and does not depend on the choice of the minimal ideal $I$.

Lemma 2. Let $(X, E)$ be a flow, let $x \in X$, and let $F \in \mathscr{S}$. Then the following statements are equivalent:

(i) $x$ is $F$-recurrent.

(ii) $c x=x$ for some idempotent $c \in F$.

(iii) $m x=x$ for some maximal idempotent $m \in F$.

Proof. Clearly (iii) implies (ii) and (ii) implies (i). Suppose $x$ is $F$-recurrent. Let $H=[r \in F \mid r x=x]$. Then $H$ is a nonempty closed semigroup, so $H$ contains an idempotent $c$. Let $m$ be a maximal idempotent in $J(F)$ with $m>c$. Then $m x=m c x=c x=x$. This proves (ii) and (iii).

It follows from the preceding lemma (or directly) that an idempotent in $F$ is an $F$-recurrent point for the action of $E$ on itself.

If $x \in X$ is an $F$-recurrent point let $J_{F}(x)=[c \in J(F) \mid c x=x]$. 
In the context of group actions, most of the following lemma and theorem are "classical."

Lemma 3. Let $(X, E)$ be a flow and let $x, y$ in $X$ be proximal. Then

(i) There is a minimal left ideal I such that $p x=p y$ for all $p \in I$.

(ii) If $y$ is an almost periodic point, then $y=u x$, for some minimal idempotent $u$.

Proof. Let $H=[p \in E \mid p x=p y]$. Then $H$ is a closed left ideal in $E(E H \subset$ $H$ ), so $H$ contains $I$, a minimal left ideal. This proves (i). Now suppose $y$ is almost periodic. Then $I y$ is a minimal set, so the set $F=[p \in I \mid p y=y]$ is a closed nonempty semigroup. Let $u$ be an idempotent in $F$. Then $u x=u y=$ $y$.

Corollary 1. The point $x$ is a distal point if and only if $u x=x$ for all minimal idempotents $u$.

Proof. Necessity is obvious. Suppose $u x=x$ for all minimal idempotents $u$. Then $x$ is an almost periodic point. If $y \in E x$ is proximal to $x$ then (since $y$ is an almost periodic point) $y=u x$ for some minimal idempotent $u$ by (ii) of Lemma 3 so $x=u x=y$.

Theorem 1. Let $E \in \mathscr{E}$ act on the compact Hausdorff space $X$ and let $x \in X$. Then the following are equivalent:

(i) $x$ is a distal point.

(ii) $\left(x, x^{\prime}\right)$ is an almost periodic point, for all almost periodic points $x^{\prime} \in X$.

(iii) If $E$ acts on $Y$ and $y \in Y$ is an almost periodic point, then $(x, y)$ is an almost periodic point for the product action of $E$ on $X \times Y$.

(iv) $c x=x$ for all idempotents $c \in E$.

(v) $u x=x$ for all minimal idempotents $u \in E$.

(vi) There is a minimal left ideal $I$ in $E$ such that $u x=x$ for all idempotents $u$ in $I$.

Suppose $F \in \mathscr{S}$ contains a minimal left ideal $I$ of $E$. (That is, $I \subset F$ and $E I \subset I$.) Then (i)-(vi) are equivalent with

(vii) $c x=x$ for all $c \in J(F)$.

(viii) $u x=x$ for all minimal idempotents $u \in J(F)$.

Proof. We first show (i) $\Rightarrow$ (iv) and (i) $\Rightarrow$ (iii). If $c \in J,(x, c x) \in P$ (the proximal relation). Thus, if $x$ is a distal point $c x=x$ for all $c \in J$, so (i) $\rightarrow$ (iv). If $x$ is a distal point and $y \in Y$ is an almost periodic point, then if $I$ is a minimal left ideal in $E$, there is an idempotent $v \in I$ with $v y=y$. Since (iv) holds, we also have $v x=x$ so $v(x, y)=(x, y)$ and $(x, y)$ is an almost periodic point. Therefore (i) $\rightarrow$ (iii). To prove that (ii) $\rightarrow($ i), suppose that $x$ is not a distal point. Then, by Corollary 1 , there is a minimal idempotent $u$ such that $y=u x \neq x$. Now $y$ is an almost periodic point but $(x, y)$ is not an almost periodic point (since $u(x, y)=(y, y) \in \Delta$, the diagonal). Now suppose (vi) holds. Then $x$ is an almost periodic point. Let $I^{\prime}$ be a minimal left ideal in $E$ and let $u^{\prime}$ be an idempotent in $I^{\prime}$. There is an idempotent $u$ in $I$ with $u \approx u^{\prime}$. Now $u x=x$, so $u^{\prime} x=u^{\prime} u x=u x=x$. That is, $u^{\prime} x=x$ for all minimal idempotents in $E$, and so by Corollary $1 x$ is a distal point. Therefore (vi) $\rightarrow$ (i). Since (iv) $\rightarrow(v),(v) \rightarrow(v i)$, and (iii) $\rightarrow$ (ii) are obvious, conditions (i)-(vi) are equivalent. 
Now suppose $F \in \mathscr{S}$ contains a minimal left ideal of $E$. Then the implications (iv) $\rightarrow$ (vii) $\rightarrow$ (viii) $\rightarrow$ (vi) are clear, and the proof is completed.

Let $F \in \mathscr{S}$, and let $(X, E)$ be a flow. A point $x$ in $X$ will be called product $F$-recurrent if, whenever $(Y, E)$ is a flow and $y \in Y$ is an $F$-recurrent point, the point $(x, y)$ is an $F$-recurrent point for the product flow $(X \times Y, E)$.

Theorem 2. Let $(X, E)$ be a flow, let $x \in X$, and let $F \in \mathscr{S}$. Then the following are equivalent:

(i) $x$ is product $F$-recurrent.

(ii) If $(Y, E)$ is a flow and $y \in Y$ is $F$-recurrent, then $J_{F}(x) \cap J_{F}(y) \neq \varnothing$.

(iii) $(x, c)$ is $F$-recurrent, for all $c \in J(F)$.

(iv) $(x, m)$ is $F$-recurrent, for all maximal idempotents $m$ in $J(F)$.

(v) $m x=x$ for all maximal idempotents $m$ in $J(F)$.

Proof. The equivalence of (i) and (ii) is immediate from Lemma 2, and since $c \in J(F)$ is a recurrent point in $E$, (i) implies (iii). Obviously (iii) $\rightarrow$ (iv). Suppose (iv) holds, and let $m \in J(F)$ be maximal. Then $(x, m)$ is $F$-recurrent so there is a maximal idempotent $m^{\prime}$ such that $m^{\prime}(x, m)=(x, m)$. Then $m^{\prime} x=x$ and $m^{\prime} m=m$. Thus $m^{\prime}>m$, so by the assumed maximality of $m$, we have $m^{\prime} \approx m, m m^{\prime}=m^{\prime}$, and $m x=m m^{\prime} x=m^{\prime} x=x$. This shows (iv) $\rightarrow$ (v).

(v) $\rightarrow$ (i). Suppose $y \in Y$ is $F$-recurrent. Let $m \in J(F)$ be maximal with $m y=y$. Then $m x=x$ so $m(x, y)=(x, y)$ and $(x, y)$ is $F$-recurrent.

Note that a distal point is product $F$-recurrent (for any $F \in \mathscr{S}$ ). For, if $y$ is an $F$-recurrent point, $c y=y$ for some $c \in J(F)$ (Lemma 2). Since $x$ is a distal point, $c x=x, c(x, y)=(x, y)$, so $(x, y)$ is $F$-recurrent.

It is natural to ask whether product $F$-recurrence characterizes distal points. (Equivalently, if $m x=x$ for every maximal idempotent $m \in J(F)$, is $c x=x$ for every $c \in J(E)$ ?) A proof that this is indeed the case for actions of $\mathbb{Z}$ or $\mathbb{N}$ (and $F=\beta \mathbb{N} \backslash \mathbb{N}$ as above) is given in [3, Theorem 9.11, p. 181]. We will prove a more general result below.

For the time being, we still consider actions of an arbitrary semigroup $E$, and obtain a sufficient condition for a product $F$-recurrent point to be a distal point. If $S$ is a semigroup, a subsemigroup $S^{*}$ of $S$ is called a cancellation semigroup if whenever $s \in S, s \notin S^{*},(s \neq e)$ then $s S^{*} \cap S^{*}=\varnothing$.

Lemma 4. Let $E \in \mathscr{E}$ and let $E^{*}$ be a nonempty closed cancellation semigroup in $E$. Then $E^{*}$ contains a maximal idempotent of $E$.

Proof. Let $c$ be an idempotent in $E^{*}$. Let $m$ be a maximal idempotent in $E$ such that $m>c$. Then $c=m c \in m E^{*} \cap E^{*}$. That is, $m E^{*} \cap E^{*} \neq \varnothing$, so $m \in E^{*}$.

Let $(X, E)$ be a flow, let $F \in \mathscr{S}$, and let $x \in X$. We say that $c \in$ $J(F)$ satisfies the cancellation semigroup condition at $x$ if there is a closed cancellation semigroup $F^{*}$ with $F^{*} \subset F$ such that $r x=c x$ for all $r \in F^{*}$. We say that $F$ satisfies the cancellation semigroup condition at $x$ if all $c \in J(F)$ satisfy the cancellation semigroup condition at $x$. In this case we write " $c$ (or $F$ ) satisfies the $C S G$ condition at $x "$. 
Corollary 2. Let $(X, E)$ be a flow, let $F \in \mathscr{S}$, and let $x \in X$.

(i) Let $c \in J(F)$ satisfy the CSG condition at $x$. Then there is a maximal idempotent $m$ of $F$ such that $m x=c x$. (It is not asserted that $m>c$.)

(ii) Suppose $F$ satisfies the CSG condition at $x$. Then $[c x \mid c \in J(F)]=$ $[m x \mid m \in J(F), m$ maximal $]$.

Corollary 3. Let $(X, E)$ be a flow, let $x \in X$, and let $F_{1}, F_{2} \in \mathscr{S}$ with $F_{1} \subset$ $F_{2}$.

(i) If $x$ is $F_{1}$ recurrent, then $x$ is $F_{2}$ recurrent.

(ii) Suppose $F_{2}$ satisfies the CSG condition at $x$ and that $x$ is product $F_{2}$ recurrent. Then $x$ is product $F_{1}$ recurrent.

Proof. The proof of (1) is obvious. Suppose that $x$ is product $F_{2}$ recurrent. Then, by Theorem 2, $m x=x$ for all maximal $m \in J\left(F_{2}\right)$. By (ii) of Corollary $1, c x=x$ for all $c \in J\left(F_{2}\right)$. Since $J\left(F_{1}\right) \subset J\left(F_{2}\right)$, we have $m x=x$ for all maximal $m \in J\left(F_{1}\right)$, and $x$ is $F_{1}$ product recurrent.

Our next corollary says that as soon as $F$ is "large enough" a product $F$ recurrent point is a distal point (so all "sufficiently large" $F$ 's have the same collection of product $F$-recurrent points). The proof follows immediately from Theorems 1 and 2, and Corollary 1.

Corollary 4. Let $(X, E)$ be a flow, let $F \in \mathscr{S}$, and let $x \in X$ be a product $F$ recurrent point. Suppose $F$ satisfies the CSG condition at $x$, and also contains a minimal left ideal of $E$. Then $x$ is a distal point of $(X, E)$.

Thus there are two properties which together guarantee that a product $F$ recurrent point is a distal point-the cancellation semigroup condition, and the condition that $F$ contain a minimal left ideal of $E$. We first consider the cancellation semigroup condition, and discuss two cases where it is satisfied. These arise from actions of finite subsets of the natural numbers (explained below) and actions of discrete abelian groups. The proofs for these two cases have certain similarities, so there will be some repetition in the arguments. It may be that a unified treatment is possible, but we prefer to deal with them separately.

\section{2. $\mathscr{F}$ ACTIONS}

Let $\mathscr{F}$ denote the collection of nonempty finite subsets of the natural numbers $\mathbb{N}$. With respect to the operation of union, $\mathscr{F}$ is a semigroup, and if we endow $\mathscr{F}$ with the discrete topology, then union extends to an associative multiplication on $\beta \mathscr{F}$ (the Stone-Čech compactification of $\mathscr{F}$ ) for which the maps $r \rightarrow r p$ are continuous.

Define a partial order on $\mathscr{F}$ by $\alpha>\delta$ if $\min \alpha>\max \delta$. For $\alpha \in \mathscr{F}$, define $\mathscr{F}_{\alpha}=[\gamma \in \mathscr{F} \mid \gamma>\alpha]$, and let $\partial \mathscr{F}=\bigcap \mathrm{cl} \mathscr{F}_{\alpha}$, where cl denotes closure in $\beta \mathscr{F}$. We call $\partial \mathscr{F}$ the boundary of $\mathscr{F}$, although it does not coincide with boundary in the topological sense. We show that $\partial F$ is a subsemigroup of $\beta \mathscr{F}$ (the "asymptotic semigroup"). To this end, fix $\alpha \in \mathscr{F}$. We first show: if $\delta>\alpha, \delta\left(\operatorname{cl} \mathscr{F}_{\alpha}\right) \subset \operatorname{cl} \mathscr{F}_{\alpha}$. For, if $\gamma_{i} \rightarrow p \in \operatorname{cl} \mathscr{F}_{\alpha}$, then $\delta \cup \gamma_{i}>\alpha$ (eventually) so $\delta \cup \gamma_{i} \rightarrow \delta p \in \operatorname{cl} \mathscr{F}_{\alpha}$. Now let $r, p \in \partial \mathscr{F}$, and let $\zeta_{i} \in \mathscr{F}$ such that $\zeta_{1} \rightarrow r$. Then $\zeta_{i} p \rightarrow r p$, and $p \in \operatorname{cl} \mathscr{F}_{\alpha}$, so $\zeta_{i} p \in \operatorname{cl} \mathscr{F}_{\alpha}$, by the above discussion, and 
$r p=\lim \zeta_{i} p \in \operatorname{cl} \mathscr{F}_{\alpha}$. Since this holds for all $\alpha \in \mathscr{F}, r p \in \bigcap \mathscr{F}_{\alpha}=\partial \mathscr{F}$. Therefore, $\partial \mathscr{F}$ is a semigroup.

By an action of $\mathscr{F}$ on the topological space $X$, we mean a map $\alpha \rightarrow T_{\alpha}$ of $\mathscr{F}$ to the continuous maps of $X$ to itself such that if $\alpha, \delta \in \mathscr{F}$ with $\alpha \cap \delta=\varnothing$, then $T_{\alpha} T_{\delta}=T_{\alpha \cup \delta}$. Note that these conditions imply that the family of transformations $\left\{T_{\alpha}\right\}$ is commutative. We just write $\alpha x$ for $T_{\alpha} x$, so $\alpha(\delta x)=(\alpha \cup \delta) x$, when $\alpha \cap \delta=\varnothing$.

An action of $\mathscr{F}$ on a (compact) space $X$ extends to an action of the asymptotic semigroup $\partial \mathscr{F}$ on $X$. Of course all elements of $\beta \mathscr{F}$ define maps of $X$ to itself. Now let $\alpha \in \mathscr{F}, q \in \partial \mathscr{F}$, and let $\left\{\zeta_{i}\right\}$ be a net in $\mathscr{F}$ with $\zeta_{i} \rightarrow q$. Then $\alpha \cap \zeta_{i}=\varnothing$ (eventually) so $\alpha\left(\zeta_{i} x\right)=\left(\alpha \cup \zeta_{i}\right) x$ and $\alpha(q x)=(\alpha q) x$ (recall that $\alpha$ defines a continuous map of $X$ ). Now suppose $\alpha_{i} \rightarrow p$ so $\alpha_{i}(q x)=\left(\alpha_{i} q\right) x$ and $p(q x)=(p q) x$. Therefore an action of $\partial \mathscr{F}$ on $X$ is defined and $(X, \partial \mathscr{F})$ is a flow. (Since $\partial \mathscr{F}$ inherits the properties of $\beta \mathscr{F}$, the maps $p \rightarrow p x$ are continuous.)

Now we show that if $X$ is compact metric space, $\partial \mathscr{F}$ has the required cancellation semigroups. (In this case $\partial \mathscr{F}$ plays the role of both $E$ and $F$.)

Lemma 5. Let $\mathscr{F}$ act on the compact metric space $X$, let $c$ be an idempotent in $\partial \mathscr{F}$, and let $x \in X$. Then there is a closed cancellation semigroup $F^{*}$ in $\partial \mathscr{F}$ such that $F^{*} x=\{c x\}$ and $F^{*} c x=\{c x\}$ (that is, if $r^{*} \in F^{*}, r^{*} x=c x$ and $\left.r^{*} c x=c x\right)$.

Proof. Let $\left\{\varepsilon_{n}\right\}$ be a sequence of positive numbers tending monotonically to 0 . We show for every $i \in \mathbb{N}$ there is a $\rho_{i} \in \mathscr{F}$ satisfying: if $i<j$ then $\rho_{i}<\rho_{j}$ and if $i_{1}<\cdots<i_{k}$, then

and

$$
d\left(\left(\rho_{i_{1}} \cup \cdots \cup \rho_{i_{k}}\right) x, c x\right)<\varepsilon_{i_{1}}
$$

$$
d\left(\left(\rho_{i_{1}} \cup \cdots \cup \rho_{i_{k}}\right) c x, c x\right)<\varepsilon_{i_{1}} .
$$

Since $c \in \partial \mathscr{F}$, there is a net $\left\{\gamma_{j}\right\}$ in $\mathscr{F}$ with $\gamma_{j} \rightarrow c$, so $\gamma_{i} x \rightarrow c x$ and $\gamma_{i} c x \rightarrow c c x=c x$. Let $\rho_{1} \in \mathfrak{F}$ such that $\rho_{1}>\{1\}, d\left(\rho_{1} x, c x\right)<$ $\varepsilon_{1}$ and $d\left(\rho_{1} c x, c x\right)<\varepsilon_{1}$. Next, let $\rho_{2}>\rho_{1}$ such that $d\left(\rho_{2} x, c x\right)<\varepsilon_{2}$, $d\left(\rho_{2} c x, c x\right)<\varepsilon_{2}, d\left(\rho_{2} \rho_{1} x, c x\right)<\varepsilon_{1}$ and $d\left(\rho_{2}\left(\rho_{1} c x\right), c x\right)<\varepsilon_{1}$ (equivalently, $d\left(\left(\rho_{1} \cup \rho_{2}\right) x, c x\right)<\varepsilon_{1}$ and $\left.d\left(\left(\rho_{1} \cup \rho_{2}\right) c x, c x\right)<\varepsilon_{1}\right)$. Note that if we put $\rho_{2}=c$, the above four inequalities certainly hold (since $\left.\rho_{1} c=c \rho_{1}\right)$ so they can be achieved by choosing $\rho_{2}>\rho_{1}$ and $\rho_{2}$ sufficiently close to $c$. Inductively, suppose $s \in \mathbb{N}$ such that, whenever $i_{1}<\cdots<i_{t}<s$ we have $\rho_{i_{j}} \in \mathscr{F}$ such that $\rho_{i_{1}}<\rho_{i_{2}}<\cdots<\rho_{i_{t}}$ with $d\left(\left(\rho_{i_{1}} \cup \cdots \cup \rho_{i_{t}}\right) x, c x\right)<\varepsilon_{i_{1}}$ and $d\left(\left(\rho_{i_{1}} \cup \cdots \cup \rho_{i_{t}}\right) c x, c x\right)<\varepsilon_{i_{1}}$. Now choose $\rho_{s} \in \mathscr{F}$ such that $\rho_{s}>\rho_{i_{t}}$ and such that $d\left(\left(\rho_{i_{1}} \cup \cdots \cup \rho_{i_{j}}\right) \rho_{s} x, c x\right)<\varepsilon_{i_{1}}, d\left(\left(\rho_{i_{1}} \cup \cdots \cup \rho_{i_{j}}\right) \rho_{s} c x, c x\right)<\varepsilon_{i_{1}}$, for all $j \leq t, d\left(\rho_{s} x, c x\right)<\varepsilon_{s}$ and $d\left(\rho_{s} c x, c x\right)<\varepsilon_{s}$. Just as in the determination of $\rho_{2}$ above, $\rho_{s}$ can be chosen in an appropriate neighborhood of $c$, since all of the inequalities hold for $\rho_{s}=c$.

If $\gamma=\left\{i_{1}, \ldots, i_{k}\right\} \in \mathscr{F}$, put $\rho_{\gamma}=\rho_{i_{1}} \cup \cdots \cup \rho_{i_{k}}$ and let $\mathscr{L}=\left[\rho_{\gamma} \mid \gamma \in \mathscr{F}\right]$. Note that if $\delta \in \mathscr{F}$ with $\delta \cap \rho_{\gamma}=\varnothing$ and $\rho_{\gamma} \cup \delta \in \mathscr{L}$, then $\delta=\rho_{\sigma}$, for some $\sigma \in \mathscr{F}$. (This remark is crucial for the proof below.)

Let $\mathscr{L}_{\alpha}=\left[\rho_{\gamma} \mid \gamma>\alpha\right]=\left[\rho_{\gamma} \mid \gamma \in \mathscr{F}_{\alpha}\right]$ and let $F^{*}=\bigcap \operatorname{cl} \mathscr{L}_{\alpha} . F^{*}$ is a closed subsemigroup of $\partial \mathscr{F}$. (The proof that $F^{*}$ is a semigroup is the same as the proof that $\partial F$ is a semigroup-in fact $F^{*}$ may be regarded as the asymptotic semigroup of the action of $\mathscr{L}$-a subaction of $\mathscr{F}$-on $X$.) 
To show that $F^{*}$ is a cancellation semigroup in $\partial \mathscr{F}$, we use a fundamental property of the Stone-Čech compactification-disjoint subsets of $\mathscr{F}$ have disjoint closures in $\beta \mathscr{F}$ (recall that $\mathscr{F}$ has the discrete topology). We first show: if $\zeta \in \mathscr{F}$ with $\zeta \notin \mathscr{L}_{\alpha}$, and $r \in F^{*}$, then $\zeta r \in \operatorname{cl} \mathscr{L}_{\alpha}^{c}$. Let $\rho_{\gamma_{i}} \rightarrow r$, where $\gamma_{i}>\alpha$. We may suppose $\zeta \cap \rho_{\gamma_{1}}=\varnothing$, so $\zeta \cup \rho_{\gamma_{i}} \rightarrow \zeta r$. But since $\zeta \notin \mathscr{L}_{\alpha}$, $\zeta \cup \rho_{\gamma_{i}} \notin \mathscr{L}_{\alpha}$, as noted above. Hence $\zeta r \in \operatorname{cl} \mathscr{L}_{\alpha}^{c}$.

Now suppose $p \in \partial \mathscr{F}, p \notin F^{*}$ and $q \in p F^{*}$, so $q=p f^{*}$ for some $f^{*} \in F^{*}$. Then $p \notin \operatorname{cl} \mathscr{L}_{\alpha}$ for some $\alpha \in \mathscr{F}$. Then $\gamma_{i} \rightarrow p$, where $\gamma_{i} \notin \mathscr{L}_{\alpha}$, so $\gamma_{i} f^{*} \rightarrow p f^{*}$. But $\gamma_{i} f^{*} \in \operatorname{cl} \mathscr{L}_{\alpha}^{c}$ by the previous paragraph, so $p f^{*} \notin \operatorname{cl} \mathscr{L}_{\alpha}$. Therefore $q=p f^{*} \notin F^{*}$. That is, if $p \notin F^{*}, p F^{*} \cap F^{*}=\varnothing$, and $F^{*}$ is a cancellation semigroup.

\section{Commutative group actions}

Let $G$ be an infinite discrete abelian group acting on a compact metric space $X$, and let $E=\beta G \backslash G$. This action of $G$ defines an action of $E$ on $X$, in the sense of our earlier discussion. (That is, $(X, E)$ is a flow.)

Let $H$ be an infinite subsemigroup of $G$ and let $F=\beta H \backslash H$. Then $F$ is a closed subsemigroup of $E$. Let $x \in X$ and let $c \in J(F)$. As in the case of $\mathscr{F}$ actions, we want to show the existence of a maximal idempotent $m$ in $F$ such that $m x=c x$. If $c x=x$, then by Lemma 2, there is a maximal idempotent $m$ in $F$ such that $m x=x=c x$. Hence we may assume that $c x \neq x$.

At this point we recall the notion of an IP set in a (multiplicative) abelian group (or semigroup) $G$. Let $\left\{q_{1}, q_{2}, \ldots\right\}$ be a sequence in $G$. The $I P$ set based on this sequence is the set $Q$ of finite products $q_{i_{1}} q_{i_{2}}, \ldots, q_{i_{h}}$. (Note that the $q_{j}$ may be repeated but the subscripts in the products defining the elements of $Q$ are not repeated.) If $\alpha=\left\{i_{1}, \ldots, i_{n}\right\} \in \mathscr{F}$ we write $q_{\alpha}$ for the product $q_{i_{1}} \cdots q_{i_{n}}$.

Let $\varepsilon_{n}$ be a decreasing sequence of positive numbers tending to 0 . If $\alpha \in \mathscr{F}$, $\alpha=\left\{i_{1}, \ldots, i_{n}\right\}$ with $i_{1}<\cdots<i_{n}$, let $\varepsilon_{\alpha}=\varepsilon_{i_{1}}$. We first show that there is an $I P$ set $Q=\left\{q_{\alpha}\right\}$ in $H$ such that $d\left(q_{\alpha} x, c x\right)<\varepsilon_{\alpha}$ and $d\left(q_{\alpha} c x, c x\right)<\varepsilon_{\alpha}$. The proof is essentially the same as the first part of the proof of Lemma 5 (and in fact the action of $Q$ on $X$ is an example of an $\mathscr{F}$ action). We define the generators of the $I P$ set $Q$ as follows. Let $q_{1} \in H$ such that $d\left(q_{1} x, c x\right)<\varepsilon_{1}$ and $d\left(q_{1} c x, c x\right)<\varepsilon_{1}$. If $q_{1}, \ldots, q_{n-1}$ have been chosen, choose $q_{n}$ in $H$ so that $d\left(q_{n} x, c x\right)<\varepsilon_{n}, d\left(q_{n} c x, c x\right)<\varepsilon_{n}, d\left(q_{\alpha} q_{n} x, c x\right)<$ $\varepsilon_{\alpha}$, and $d\left(q_{\alpha} q_{n} c x, c x\right)<\varepsilon_{\alpha}$ for all subsets $\alpha$ of $\{1,2, \ldots, n-1\}$. (If $q_{n}$ is replaced by $c$ in these four inequalities, the inequalities obviously hold, so $q_{n}$ can be chosen in a neighborhood of $c$ so that they still hold.) Now let $Q_{\alpha}=\left[q_{\beta} \mid \beta>\alpha\right]=\left[q_{\beta} \mid \beta \in \mathscr{F}_{\alpha}\right]$ and let $\partial Q=\bigcap \mathrm{cl} Q_{\alpha}$, where the closure is taken in $\beta G$. Just as in the case of $\mathscr{F}$ it is shown that $\partial Q$ is a semigroup. Moreover it is clear that if $p \in \partial Q, p x=p c x=c x$. Also, since $H$ is a semigroup, all $q_{\alpha}$ are in $H$, so $\partial Q \subset \beta H$. In fact, $\partial Q \subset \beta H \backslash H=F$. For, let $p \in \partial Q$ and let $q_{\alpha} \rightarrow p$. If $p \in H$, then since $G$ is discrete (a subnet of) $q_{\alpha}=q \in H$. Then we would have $q x=c x, q q x=q c x=c q x=c c x=c x=q x$, so $q x=x$ and $c x=q x=x$, contrary to our standing assumption. However, it is apparently not necessarily the case that $\partial Q$ is a cancellation semigroup. In order to achieve this, it is necessary to "refine" the $I P$ set $Q$. To this end, we say that an $I P$ set $Q=\left\{q_{\alpha}\right\}$ is $R I P$ ( $R$ is for "rare") if whenever $g \in G$, 
$g \notin Q_{\alpha}$ (with $g \neq e$ ) then there is a $\beta^{*} \subset \mathscr{F}\left(\beta^{*}=\beta^{*}(\alpha, g)\right.$ ) such that if $\beta>\beta^{*}$, we have $g q_{\beta} \notin Q_{\alpha}$ (equivalently $g Q_{\beta^{*}} \cap Q_{\alpha}=\varnothing$ ).

Lemma 6. If $Q$ is $R I P$ then $\partial Q$ is a cancellation semigroup.

Proof. Here also the proof resembles the proof for $\mathscr{F}$. We first show: if $g \in G$ with $g \neq e, g \notin Q_{\alpha}$, and $r \in \partial Q$ then $g r \notin \mathrm{cl} Q_{\alpha}$. Let $\left\{q_{\beta_{i}}\right\}$ be a net in $Q$ with $q_{\beta_{i}} \rightarrow r$. We may suppose $\beta_{i}>\beta^{*}=\beta^{*}(\alpha, g)$. Then $g q_{\beta_{i}} \notin Q_{\alpha}$. Thus $g q_{\beta_{i}} \in G \backslash Q_{\alpha}$ and $g q_{\beta_{i}} \rightarrow g r$, so $g r \in \operatorname{cl}\left(G \backslash Q_{\alpha}\right)$. Since disjoint subsets of $G$ have disjoint closures in $\beta G$, we have $g r \notin \mathrm{cl} Q_{\alpha}$.

Now let $p \in \beta G$ with $p \notin \partial Q$, and let $r \in \partial Q$. We show $p r \notin \partial Q$. Since $p \notin \partial Q, p \notin \operatorname{cl} Q_{\alpha}$, for some $\alpha \in \mathscr{F}$. Then there is a net $\left\{g_{j}\right\}$ in $G$ with $g_{j} \rightarrow p$ and $g_{j} \notin Q_{\alpha}$, so $g_{j} r \notin \mathrm{cl} Q_{\alpha}$, by the first part of this proof. Therefore $g_{j} r \in \operatorname{cl}\left(G \backslash Q_{\alpha}\right), p r \in \operatorname{cl}\left(G \backslash Q_{\alpha}\right)$ so $p r \notin \operatorname{cl}\left(Q_{\alpha}\right)$. It follows that $p r \notin \partial Q$.

Now we show that an IP set can always be "refined" to an RIP set. We first suppose that the group $G$ is countable. Let $Q=\left\{q_{\alpha}\right\}$ be an $I P$ set in $G$ with infinite range (that is, the cardinality of the set $\left\{q_{\alpha} \mid \alpha \in \mathscr{F}\right\}$ is infinite). We suppose, without loss of generality, that $q_{1}=e$, and we construct $R=\left\{r_{\alpha}\right\}$ an $R I P$ set with infinite range such that range $R \subset$ range $Q . R$ will be defined by its generators $r_{1}, r_{2}, \ldots$ Let $G=\left\{g_{1}=e, g_{2}, \ldots\right\}$. Let $r_{1}=e$. Let $r_{2}=q_{\alpha_{2}}$ where $q_{\alpha_{2}} \neq e$ or $g_{2}^{ \pm 1}$. If $r_{1}, \ldots, r_{n-1}$ have been defined, $r_{j}=q_{\alpha_{j}}$, let $r_{n}=q_{\alpha_{n}}$ where $\alpha_{n}>\alpha_{n-1}$ and $q_{\alpha_{n}} \neq g_{a}^{ \pm 1} r_{i_{1}} \cdots r_{i_{t}} r_{j_{1}}^{-1} \cdots r_{j_{v}}^{-1}$ where $a \leq n$, $1 \leq i_{1}<\cdots<i_{t}<n, 1 \leq j_{1}<\cdots<j_{v}<n$. We show that $R=\left\{r_{\alpha}\right\}$ is $R I P$. Let $g=g_{n} \in G(n>1)$. Let $\beta^{*} \in \mathscr{F}, \beta^{*}=\left\{s_{1}, \ldots, s_{k}\right\}$ with $s_{1}<\cdots<s_{k}$ and $s_{1}>n$. We show $g R_{\beta} \cap \cap R_{\alpha}=\varnothing$. Suppose we have an equation $g_{n} r_{\zeta}=r_{\nu}$, $r_{\zeta} \in R_{\beta^{*}}, r_{\nu} \in R_{\alpha}$. If $r_{\zeta}=r_{a_{1}} \cdots r_{a_{k}}, r_{\nu}=r_{b_{1}} \cdots r_{b_{l}} \quad\left(n<a_{1}<\cdots<a_{k}\right.$, $\left.b_{1}<\cdots<b_{l}\right)$ then $g_{n} r_{a_{1}} \cdots r_{a_{k}}=r_{b_{1}} \cdots r_{b_{l}}$. Cancel any terms in common. If all the terms on the left cancel, then $g_{n} \in R_{\alpha}$ contary to hypothesis. Otherwise, we may suppose $r_{a_{i}} \neq r_{b_{j}}$. Suppose $a_{k}$ is the largest subscript appearing. Then solving for $r_{a_{k}}$ we obtain $r_{a_{k}}=g_{n}^{-1} r_{b_{1}} \cdots r_{b_{l}} r_{a_{1}}^{-1} \cdots r_{a_{k-1}}^{-1}$ which contradicts the definition of $R$ (since $a_{k}>n$ ). A similar contradiction is reached if the maximum subscript is $b_{l}$ (since in this case $b_{l}>a_{k}>n$.)

Now we remove the countability restriction. As above, let $Q=\left\{q_{\alpha}\right\}$ be an $I P$ set in $G$ and let $\Sigma$ be the (countable) group generated by $Q$. As in the preceding paragraph, refine $Q$ to $R$, which is an $R I P$ set with respect to the group $\Sigma$. Now if $g \in G \backslash \Sigma, g \neq e$, then obviously $g R \cap R=\varnothing$, so in fact $R$ is an $R I P$ set with respect to the group $G$.

Summarizing, if $F=\beta H \backslash H$, and $c \in J(F)$ with $c x \neq x$, we have shown that there is an $R I P$ subset $R$ of $H$ such that $p x=c x$ for all $p \in \partial R$. Moreover $\partial R$ is a cancellation semigroup (whenever $R$ is $R I P$ ). Hence we may apply Corollary 2, which guarantees the existence of a maximal idempotent $m$ in $F$ such that $m x=c x$. (As noted above, if $c x=x$, such an $m$ always exists.)

Now we turn to the question of when $F$ contains a minimal left ideal of $E$. For actions of $\mathscr{F}$, the finite subsets of $\mathbb{N}$, we have $E=F=\partial \mathscr{F}$, so there is nothing to prove. Our next lemma gives necessary and sufficient conditions in the case of group actions. As above, $E=\beta G \backslash G, F=\beta H \backslash H$, where $G$ is an infinite discrete abelian group and $H$ is a subsemigroup of $G$. First, we require a lemma. 
Lemma 7. $G E \subset E$ and $H F \subset F$.

Proof. The first containment is clear. Now $H F \subset G E \subset E$. If $h \in H, f \in F$, and $\left\{k_{i}\right\}$ is a net in $H$ such that $k_{i} \rightarrow f$, then $h k_{i} \rightarrow h f, h k_{i} \in H$, so $h f \in \beta H \backslash G \subset \beta H \backslash H=F$.

Lemma 8. The following are equivalent:

(i) $F$ contains a minimal left ideal of $E$.

(ii) $E f \subset F$ for some $f \in F$.

(iii) $G=H H^{-1}$.

Proof. (i) $\rightarrow$ (ii). Suppose $F \supset I$, a minimal left ideal of $E$. Then, if $f \in I$, $E f \subset E I \subset I \subset F$.

(ii) $\rightarrow$ (i). Let $I$ be a minimal left ideal of $E$. Then $I f$ is a closed ideal of $E$, so $I f \supset K$, a minimal left ideal of $E$. Then $K \subset I f \subset E f \subset F$.

(iii) $\rightarrow$ (ii). If $h_{1}, \ldots, h_{n} \in H$, then (since $G$ is abelian) $h_{1} F \cap \cdots \cap h_{n} F \supset$ $h_{1} \cdots h_{n} F \neq \varnothing$, so the collection $\{h F\}_{h \in H}$ has the finite intersection property. Let $f^{*} \in \bigcap_{h \in H} h F$. Now let $g \in G=H H^{-1}$, so $g=k h^{-1}(k, h \in H)$, and $g f^{*}=k h^{-1} f^{*} \in k F \subset F$. Therefore $G f^{*} \subset F$, and it follows that $E f^{*} \subset F$.

(ii) $\rightarrow$ (iii). Let $G^{\prime}=[g \in G \mid g f \in F]$. We first note that $G^{\prime} \subset H H^{-1}$. If not, let $g \in G^{\prime}$ with $g \notin H H^{-1}$ so $g H \cap H=\varnothing$. Then (since disjoint subsets of $G$ have disjoint closures in $\beta G) \quad g F \cap F \subset g \bar{H} \cap \bar{H}=\overline{g H} \cap \bar{H}=\varnothing$ which contradicts $g f \in F$. Next, we show that $G \backslash G^{\prime}$ is finite. If not, there is a net $\left\{g_{i}\right\}$ in $G \backslash G^{\prime}$ such that $g_{i} \rightarrow \eta \in E$. Now $g_{i} f \in E$ and $g_{i} f \notin F$, so $g_{i} f \in \overline{G \backslash H}$, and $\eta f \in \overline{G \backslash H}$. Therefore $\eta f \notin \bar{H}=F$, which contradicts $E f \subset F$. $G$.

Thus $G^{\prime} \subset H H^{-1}$, so $H H^{-1}$ is cofinite. But $H H^{-1}$ is a group, so $H H^{-1}=$

We may summarize our conclusions as follows.

Theorem 3. Let $(X, E)$ be a flow, where $X$ is a compact metric space, and $E=\partial \mathscr{F}$ or $E=\beta G \backslash G$ ( $G$ a discrete abelian group). Let $F$ (respectively) be $\partial \mathscr{F}$ or $\beta H \backslash H$, where $H$ is a subsemigroup of $G$. Let $x \in X$. Then $[c x \mid c \in J(F)]=[m x \mid m \in J(F)$ with $m$ maximal $]$.

Theorem 4. In addition to the hypotheses of Theorem 3, suppose (in case $E=$ $\beta G \backslash G$ and $F=\beta H \backslash H)$ that $G=H H^{-1}$. Then the following are equivalent:

(i) $x$ is a distal point.

(ii) $x$ is $F$-product recurrent.

(iii) $u x=x$ for all minimal idempotents in $J(F)$.

(iv) $m x=x$ for all maximal idempotents in $J(F)$.

(v) $c x=x$ for all $c \in J(F)$.

If $G$ is the additive group $\mathbb{R}^{n}$ or $\mathbb{Z}^{n}$ and $H$ is a cone with nonempty interior, then (iii) of Lemma 8 is valid. It follows that in this case the notion of product $F$-recurrence is independent of the cone $H$.

Note that (in contrast with condition (ii) in Theorem 1) we cannot assert that a point $x$ in a flow $X$ for which $\left(x, x^{\prime}\right)$ is recurrent for all $x^{\prime} \in X$ is a distal point. This is because of the existence of "rigid" flows which are not distal [6, 7]. 
Finally, returning to the consideration of actions of arbitrary semigroups $E$, a general question is to characterize the closed semigroups $F \in \mathscr{S}$ for which a product $F$-recurrent point is a distal point. Another question (even for $\mathbb{Z}$ or $\mathbb{N}$ actions): If $(x, y)$ is recurrent for all almost periodic points $y$, is $x$ necessarily a distal point?

We would like to thank Ken Berg for useful discussions; in particular, he suggested condition (iii) in Lemma 8.

We would also like to thank David Ellis for pointing out a mistake in our original construction of $R I P$ sets.

\section{REFERENCES}

1. J. Auslander, Minimal flows and their extensions, North-Holland Math. Studies, Notas de Matematicas 122, North-Holland, 1988.

2. R. Ellis, Lectures in topological dynamics, Benjamin, 1969.

3. H. Furstenberg, Recurrence in ergodic theory and combinatorial number theory, Princeton Univ. Press, 1981.

4. _ , IP systems in ergodic theory, Conference in Modern Analysis and Probability, Contemporary Math., vol. 26, Amer. Math. Soc., Providence, RI, 1984, pp. 131-148.

5. H. Furstenberg and B. Weiss, The finite multipliers of infinite ergodic transformations, Structure of Attractors in Dynamical Systems, Lecture Notes in Math., vol. 668, Springer, 1978, pp. 127-133.

6. S. Glasner and D. Maon, Rigidity in topological dynamics, Ergodic Theory Dynamical Systems 9 (1989), 309-320.

7. Y. Katznelson and B. Weiss, When all points are recurrent/generic, Progress in Math., vol. 10, (Proc. Special Year, Maryland 1979-1980), Birkhaüser Boston, 1981, pp. 195-210.

Department of Mathematics, University of Maryland at College Park, College PARK, MARYLAND 20742

Department of Mathematics, Hebrew University, Jerusalem, Israel 